'Sección Reumatología, Hospital del Salvador, Universidad de Chile.

${ }^{2}$ Servicio de Anatomía patológica, Hospital del Salvador.

${ }^{3}$ Sección Hematología, Hospital del Salvador.

${ }^{4}$ Servicio de Medicina, Hospital del Salvador.

Sin Fuente de apoyo financiero Autores declaran no tener conflictos de interés.

Recibido el 14 de agosto de 2017, aceptado el 15 de mayo de 2018.

Correspondencia a: Daniel Erlij

Hospital del Salvador, Avenida Salvador 364, Providencia, Santiago, Chile. danerlij@gmail.com

\section{Oligoartritis, paniculitis y pioderma gangrenoso como manifestaciones de un Linfoma no Hodgkin}

\author{
DANIEL ERLIJ ${ }^{1}$, CAROLINA CUELLAR $^{1}$, ANGELA RIVERA ${ }^{1}$, \\ NATALIA BADILLA ${ }^{1}$, NICIA MOLDENHAUER ${ }^{1}$, CAROLINA FOSTER ${ }^{1}$, \\ BERNARDO LÓPEZ ${ }^{2}$, XIMENA VALLADARES $^{3}$, MARY ANN STEVENS $^{4}$
}

\section{Oligoarthritis, panniculitis and pyoderma gangrenosum associated with non-Hodgkin lymphoma. Report of one case}

An assortment of clinical and laboratory abnormalities may occur as paraneoplastic syndromes in lymphomas. Rheumatological and dermatological manifestations such as paraneoplastic arthritis and pyoderma gangrenosum must be underscored. We report a 28 years old woman who developed pyoderma gangrenosum and two years later presented with arthritis of knees and ankles associated with panniculitis interpreted as erythema induratum that was pathologically confirmed. She developed a reactivation of pyoderma gangrenosum, that was refractory to treatment. Complementary studies showed a pulmonary nodule and a right paravertebral mass with involvement of the psoas muscle. Biopsies of both masses and a new pathological skin study demonstrated a large $B$-cell non-Hodgkin's lymphoma.

(Rev Med Chile 2018; 146: 534-537)

Key words: Arthritis; Lymphoma, Non-Hodgkin; Panniculitis; Parancoplastic Syndromes; Pyoderma Gangrenosum.

\section{L}

os síndromes paraneoplásicos relacionados a linfomas, incluyen expresiones clínicas de varias áreas de la medicina, destacando las manifestaciones reumatológicas como poliartritis, hematológicas como anemia hemolítica y dermatológicas como el pioderma gangrenoso. La primera aproximación a dichas entidades, consiste en determinar con claridad si el fenómeno es realmente paraneoplásico, es decir, humoral o es consecuencia de la invasión tumoral directa. En el caso de las manifestaciones reumatológicas, dicha distinción no es compleja, pero en aquellas del área dermatológica, puede resultar un difícil reto, especialmente si se considera que existen linfomas que se inician en la piel o que en su evolución pueden infiltrar tejidos cutáneos.

\section{Caso clínico}

Mujer de 28 años, antecedente de pioderma gangrenoso hace dos años, presenta cuadro de 3 meses de edema de extremidades inferiores, artritis de rodillas y tobillos, fiebre hasta $39^{\circ} \mathrm{C}$, baja peso 8 kilos y sudoración nocturna, a lo cual se suman lesiones cutáneas tipo paniculitis en cuatro extremidades (Figura 1a). En los exámenes de laboratorio destacaba hemoglobina $10 \mathrm{~g} / \mathrm{dl}$, leucocitos 3.500 $\mathrm{x} \mathrm{mm^{3 }}$, plaquetas $600.000 \mathrm{x} \mathrm{mm}^{3}$, VHS $17 \mathrm{~mm} / \mathrm{h}$, albúmina $2,3 \mathrm{~g} / \mathrm{dL}$ y resto de exámenes generales normales. La pesquisa viral (VIH, VHB, VHC) fue negativa. El estudio reumatológico demostró anticuerpos antinucleares (ANA) positivos patrón nucleolar $>1 / 160$, con ENA, factor reumatoídeo (FR), ANCA y DNA negativos, pero complemento 


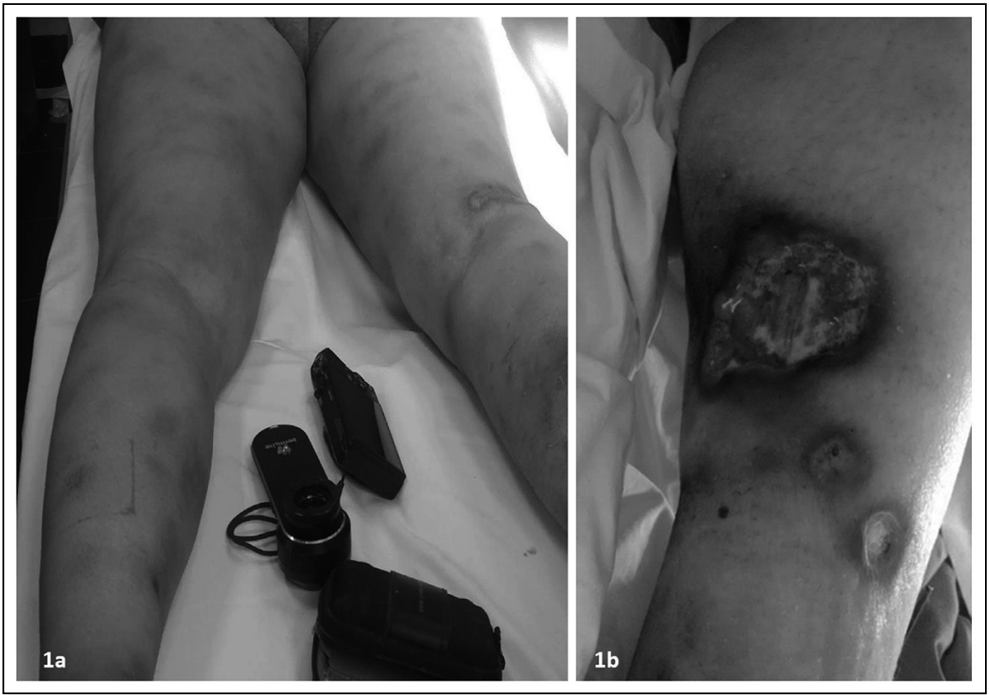

Figura 1. A: Vista posterior de extremidades inferiores de la paciente, evidenciándose lesiones paniculíticas. B: Reactivación de pioderma gangrenoso a partir del sitio de toma de biopsia en cara posterior de extremidad inferior izquierda.
C3 en 63 mg/dl (normal 90-180), con C4 normal. Las lesiones cutáneas impresionan clínicamente como un eritema indurado de Bazin, realizándose en consecuencia, estudio de Mycobacterium tuberculosis (ELISPOT, baciloscopías y cultivos de lavado bronquioalveolar) que resulta negativo. Sin embargo, el estudio imagenológico con tomogra- fía computada evidenció nódulo pulmonar de 2 $\mathrm{cm}$, de centro hipodenso, en relación al plano de la cisura menor, además de múltiples nódulos en el tejido celular subcutáneo (Figura $2 \mathrm{a}$ y $2 \mathrm{~b}$ ). La biopsia cutánea de las lesiones mostró paniculitis mixta de predominio lobulillar, con vasculitis focal (vasculitis nodular). La paciente evoluciona

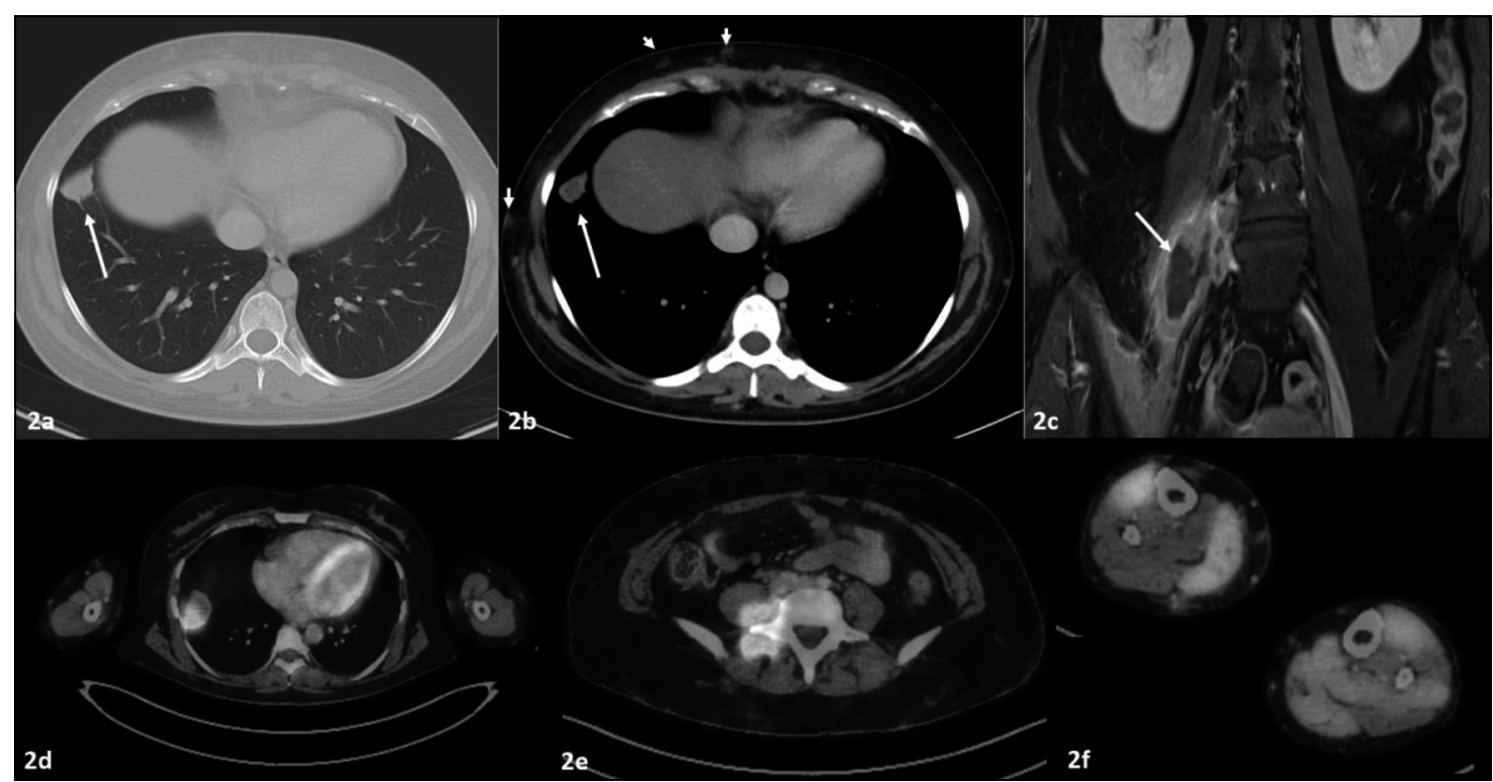

Figura 2. A: $C T$ de tórax ventana pulmonar que muestra nódulo pulmonar de $2 \mathrm{~cm}$. B: $\mathrm{CT}$ ventana mediastínica que muestra además nódulos subcutáneos (flechas pequeñas). C: Resonancia secuencia T1 con gadolinio que evidencia masa de 7,3 cm con realce periférico, paravertebral derecha con compromiso del psoas. D, E y F: PETCT que demuestra lesiones ya descritas hipermetabólicas. 


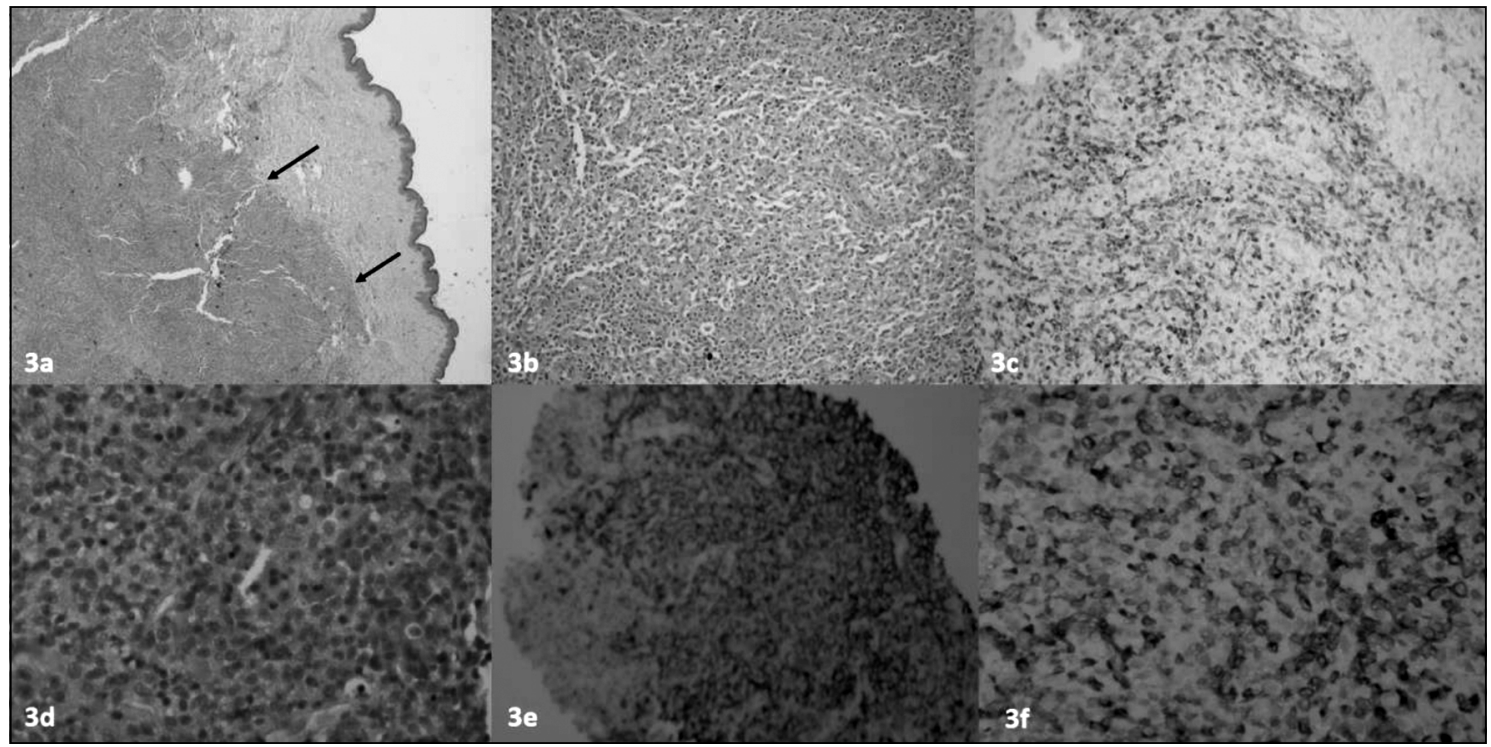

Figura 3. A: Histología de piel con infiltrado dérmico de células neoplásicas (4x, HE). B: células neoplásicas a nivel de dermis con mayor aumento (20x, HE). C: Inmunohistoquímica de células descritas con positividad CD20 (20x). D: Histología nódulo pulmonar que muestra células grandes de citoplasma claro y núcleos prominentes con nucléolos (40x HE). E: Inmunohistoquímica de células descritas con positividad CD20 (10X). F: Inmunohistoquímica con positividad BCL2 para células de nódulo pulmonar (40X).

con reactivación de pioderma gangrenoso a partir del sitio de biopsia (Figura 1b), sin respuesta a tratamiento local con inyecciones esteroidales perilesionales ni sistémico con prednisona y metotrexato, sumándose clínicamente paresia de extremidad inferior derecha. La resonancia magnética lumbar evidenció masa paravertebral derecha a nivel de L5 con compromiso del psoas (Figura 2c). PETCT muestra hipermetabolismo a nivel de nódulos subcutáneos, nódulo pulmonar y masa paravertebral (Figura 2d, 2e y 2f). Finalmente, se realiza biopsia de los 3 sitios descritos, las cuales mostraron un linfoma no Hodgkin (LNH) de células grandes $B$ (Figuras 3a-3f). Se inicia esquema R-CHOP, siguiendo con esquemas de segunda y tercera línea, con refractariedad a tratamiento y disminución parcial de los fenómenos paraneoplásicos. La paciente se trata finalmente en cuidados paliativos.

\section{Discusión}

Los trastornos linfoproliferativos están asociados a varios síndromes paraneoplásicos, que pueden ser vistos en todos los subtipos de LH y grados de LNH, principalmente de estirpe B. Son manifestaciones paraneoplásicas frecuentes las hematológicas, neurológicas y dermatológicas, destancando también aquellas reumatológicas como la artritis y vasculitis ${ }^{1}$.

La artritis paraneoplásica se caracteriza por ser un cuadro de compromiso oligoarticular o poliarticular, generalmente asimétrica. Compromete grandes articulaciones, con predominio en extremidades inferiores. Un análisis de 8 series de casos que incluían un total de 121 pacientes, mostró que la edad media del inicio de los síntomas era de 54 años, predominando el sexo masculino (relación 1,7:1) y que hasta un tercio se asociaba a neoplasia hematológica. El 27\% fue positivo para FR y $19 \%$ para ANA 2 .

Las vasculitis paraneoplásicas representan 5\% de todas las vasculitis y se asocian hasta en $63 \%$ a malignidad hematológica. El LNH es responsable de alrededor de $9 \%$ de los casos. La vasculitis más frecuente es la cutánea leucocitoclástica, seguida de las sistémicas ${ }^{3}$.

El pioderma gangrenoso constituye una dermatosis neutrofílica ulcerativa poco frecuente, asociado a enfermedad sistémica en $50-78 \%$ de 
los casos, principalmente enfermedad inflamatoria intestinal y artritis reumatoídea. Hasta $7 \%$ se relaciona a neoplasias hematológicas, principalmente a leucemia mieloide aguda. El subtipo buloso es el más comúnmente observado en este contexto. Existen escasos reportes asociados a linfomas. Respecto a la asociación entre artritis y pioderma gangrenoso, un antiguo estudio de 15 pacientes con dicha dermatosis, describió la presencia de poliartritis en 8 de ellos, de los cuales sólo 2 constituyeron una artritis reumatoídea seropositiva. Del resto, 4 presentaron un comportamiento de artritis erosiva ${ }^{5}$.

La paniculitis corresponde a un grupo de trastornos inflamatorios que afecta la grasa subcutánea, expresados como nódulos eritematosos dolorosos principalmente de extremidades inferiors, aunque también pueden afectar tronco, cara e incluso cuero cabelludo. La etiología puede ser infecciosa, neoplásica, inmunológicas, entre otras, siendo la biopsia clave para clasificarlas según predominio inflamatorio septal o lobulillar y según la presencia o no de vasculitis ${ }^{6}$. Las paniculitis, como otras enfermedades inflamatorias de la piel, constituye un proceso dinámico en el que tanto la composición como la distribución del infiltrado inflamatorio puede cambiar en el curso de unos pocos días, situación a tener en cuenta para la interpretación del estudio histológico. Los tipos más frecuentes son el eritema nodoso y el eritema indu$\mathrm{rado}^{7}$. Este último, también denominado vasculitis nodular, es conocido como eritema indurado de Bazin cuando está en relación a tuberculosis, considerándose una inmunoreacción a la infección por el mycobacterium, con mayor tendencia a la persistencia y ulceración de las lesiones. Es más frecuente en mujeres, generalmente entre 30 y 40 años ${ }^{8}$. Las paniculitis asociadas a malignidad se han descrito en relación a LH y leucemias agudas, debiendo diferenciarse en todos los casos, si el compromiso es realmente paraneoplásico o existe infiltración tumoral. Es así como existen linfomas, especialmente de estirpe $\mathrm{T}$, que comprometen primariamente la piel. Más aún, está descrita una entidad infrecuente denominada Linfoma de cé- lulas $\mathrm{T}$ similar a paniculitis, el cual demuestra al estudio histológico, un compromiso más allá del celular subcutáneo, incluso dermis profunda, lo cual no es propio de las paniculitis9.

En el caso expuesto, la oligoartritis y el pioderma gangrenoso corresponden a manifestaciones paraneoplásicas, mientras que la paniculitis debe considerarse como tumoral, a pesar de evidenciarse sólo en la segunda biopsia. Destaca en el caso, la concomitancia inusual de paniculitis con pioderma gangrenoso, lo cual hace necesario considerar la presencia de neoplasias en la evaluación de los pacientes con dichas manifestaciones.

\section{Referencias}

1. Hagler K, Lynch J. Paraneoplastic Manifestations of Lymphoma. Clinical Lymphoma 2004; 5: 29-36.

2. Manger B, Schett G. Paraneoplastic Syndromes in Rheumatology. Nature Reviews Rheumatology. 2014; 10: 662-70.

3. Wooten M, Jasin H. Vasculitis and Lymphoproliferative Diseases. Semin Arthritis Rheum 1996; 26: 564-74.

4. Ahronowitz I, Harp J, Shinkai K. Etiology and Management of Pyoderma Gangrenosum: A Comprehensive Review. Am J Clin Dermatol 2012; 13 (3): 191-211.

5. Holt PJ, Davies MG, Saunders KC, Nuki G. Pyoderma gangrenosum: clinical and laboratory findings in 15 patients with special reference to polyarthritis. Medicine (Baltimore) 1980; 59 (2): 114-33.

6. Prieto VG, Ivan D. Panniculitis: a diagnostic algorithm. Diagn Histopathol 2009; 15: 195-202.

7. Blake T, Manahan M, Rodins K. Erythema nodosum: are view of an uncommon panniculitis. Dermatol Online J 2014; 20 (4): 22376.

8. Segura S, Pujol RM, Trindade F, Requena L. Vasculitis in erythema induratum of Bazin: a histopathologic study of 101 biopsy specimens from 86 patients. J Am Acad Dermatol 2008; 59: 839-51.

9. Willemze R, Jansen PM, Cerroni L, Berti E, Santucci M, Assaf C, et al. Subcutaneous panniculitis-like T- cell lymphoma: definition, classification, and prognostic factors: an EORTC Cutaneous Lymphoma Group Study of 83 cases. Blood 2008; 111 (2): 838-45. 